\title{
Bimodal bilingualism as multisensory training?: Evidence for improved audiovisual speech perception after sign language exposure
}

\author{
Joshua T. Williams ${ }^{1,2,3,}{ }^{*}$, Dr. Isabelle Darcy ${ }^{2,5}$, Dr. Sharlene D. Newman ${ }^{1,2,4}$ \\ ${ }^{1}$ Department of Psychological and Brain Sciences, ${ }^{2}$ Program in Cognitive Science, ${ }^{3}$ Speech and \\ Hearing Sciences, ${ }^{4}$ Program in Neuroscience, and ${ }^{5}$ Second Language Studies, Indiana \\ University
}

*Corresponding Author

Joshua Williams

Address: Cognitive Neuroimaging Laboratory, Indiana University, 1101 E. $10^{\text {th }}$ Street, Bloomington, IN, 47405.

Phone: $+1-812-856-1776$

Email: willjota@indiana.edu

Keywords: American Sign Language; Audiovisual Speech; lipreading; bimodal bilingualism; longitudinal fMRI study 


\begin{abstract}
The aim of the present study was to characterize effects of learning a sign language on the processing of a spoken language. Specifically, audiovisual phoneme comprehension was assessed before and after 13 weeks of sign language exposure. L2 ASL learners performed this task in the fMRI scanner. Results indicated that L2 American Sign Language (ASL) learners' behavioral classification of the speech sounds improved with time compared to hearing nonsigners. Results indicated increased activation in the supramarginal gyrus (SMG) after sign language exposure, which suggests concomitant increased phonological processing of speech. A multiple regression analysis indicated that learner's rating on co-sign speech use and lipreading ability was correlated with SMG activation. This pattern of results indicates that the increased use of mouthing and possibly lipreading during sign language acquisition may concurrently improve audiovisual speech processing in budding hearing bimodal bilinguals.
\end{abstract}




\section{Introduction}

To understand language is to integrate multimodal information (Vigliocco, Perniss, \& Vinson, 2014). Audiovisual information is key to understanding speech, especially with hard-toperceive contrasts (Navarra \& Soto-Faraco, 2007) or ambiguity (Lidestam \& Beskow, 2006). Even sign language users require integration of facial and corporal cues for successful language processing (Bickford \& Fraychineaud, 2008). The unique separation of the articulators during the production of sign (e.g., hands) and speech (e.g., mouth) allow for simultaneous codeblending in hearing signers (Emmorey, Borinstein, Thompson, \& Gollan, 2008). That is, often hearing signers will communicate using both spoken and sign language simultaneously. With such an importance on multimodal integration for language processing and the concomitant ability to sign and speak in parallel, sign language learners may have increased audiovisual processing abilities as a consequence. In the present study, we examined how exposure to sign language facilitates opportunities for increased audiovisual processing, which in turn affects spoken language processing.

Speech processing is inherently a multimodal experience (Summerfield, 1987). Forty years of work investigating the McGurk Effect has indeed demonstrated that speech processing is not only reliant on the auditory stream, but also the visual stream (McGurk \& MacDonald, 1976; Massaro \& Simpson, 2014). Visual information is needed most when communication occurs in noisy environments (Girin, Schwartz, \& Feng, 2001; Sumby \& Pollack, 1954). Thus, typically hearing speakers use both auditory and visual information during face-to-face communication. The ability to process language audiovisually varies from speaker to speaker; however, many studies have shown enhancements in audiovisual processing (e.g., detection, discrimination, and localization) after multisensory training (Lehmann \& Murray, 2005; 
Lovelace, Stein, \& Wallace, 2003; Strelnikov, Rosito, \& Barone, 2011). With evidence of training-induced improvements in audiovisual processing, the current study explored the effect of "linguistic training" from a visual language on audiovisual processing in individuals who are learning a sign language.

Spoken and sign languages differ in terms of their input-output systems with which they are perceived and produced, despite showing similar patterns of neural activation and language network recruitment for native signers and speakers alike (Leonard et al., 2012; MacSweeney et al., 2002, 2006, 2008; Petitto et al., 2000). Spoken languages lend themselves to audiovisual integration because they require the use of oral articulators to aid in auditory processing. Sign languages, on the other hand, use the manual-visual modality in order to process language. Despite modality differences, sign languages exploit the mouth in a number of ways. For instance, sign languages use the mouth for independent morphemes that inflect verbs or noun phrases (Bickford \& Fraychineaud, 2008). Beyond grammatical uses of the mouth, the mouth plays an important role in lexical processing such that signers often borrow mouthings from spoken languages (Bickford \& Fraychineaud, 2008; Boyes-Braem \& Sutton-Spence, 2001). A commonly used example of borrowed mouthings in American Sign Language (ASL) is the simultaneous production of [f]] on the mouth during the manual sign FINISH (Bickford \& Fraychineaud, 2008; Boyes-Braem \& Sutton-Spence, 2001; Emmorey et al., 2005). Similarly, mouthings can be used to disambiguate homophonous manual signs such as signs that share the same phonological features like handshape, location, and movement (e.g., CANCEL ${ }^{1}$ and CRITICIZE), where the full English word is articulated during sign production. Often hearing bimodal bilinguals (e.g., ASL/English bilinguals) will mouth English translations of the signs they are producing (Capek, Campbell, \& Woll, 2008; Davis, 1990; Emmorey et al., 2008). The 
ability to code-blend, or sign and speak simultaneously, is afforded by the separation of the two articulatory systems. Unlike during audiovisual processing where visual information facilitates greater auditory comprehension (Summerfield, 1987), novice learners of a sign language may need borrowed mouthings in order to comprehend newly-acquired lexical items in sign language. Attunement to the mouth for lexical sign processing and the general immersive experience relying on the visual system to process language may impact spoken language audiovisual processing.

It has been well documented that the brain undergoes neuroplastic changes due to new experiences and training (Draganski et al., 2004; Schlaug et al., 2010). One manner in which neuroplasticity can be induced is through experience with a new language (Mechelli et al., 2004). Several recent studies have shown that the neural network that underlies the first language is changed during the acquisition of a second (Kovelman et al., 2008; Mei et al., 2014; Jones et al., 2012). For instance, the left inferior frontal gyrus is more involved in native language processing for bilinguals than monolinguals (Kovelman et al., 2008; Jones et al., 2012) and the activation of the native reading network is altered after semantic training in a novel language (Mei et al., 2014). If learning a new language, specifically one within one's native language modality, can alter the neural network that underlies native language processing, it would be assumed that learning a language within a new language modality can also alter underlying cognitive structures. Because bimodal bilinguals attune to the mouth during sign language processing, those areas involved in audiovisual processing may also undergo neuroplatic changes.

Audiovisual processing has been shown to activate the superior temporal sulcus (STS; Campbell, 2008; Hocking \& Price, 2008; Noesselt et al., 2007). The STS is also involved in 
biological motion processing, face perception, and is especially attuned to mouth movements that mirror speech and its auditory spatiotemporal dynamics (Campbell, 2008; McCarthy, Puce, Belger, \& Allison, 1999; Pelphrey et al., 2003). Furthermore, the STS is super-additively activated by audiovisual speech sounds when compared to visual mouth movement or audition alone (Wright et al., 2003). It is assumed that greater audiovisual processing should also lead to greater phonological processing. That is, greater audiovisual processing would entail better identification of visual phonemes. Enhanced phonemic processing is likely to also engage the supramarginal gyrus (SMG), an area implicated in phonological processing (Hartwigsen et al., 2010; Sliwinska et al., 2012; Stoeckel et al., 2009). Ruytjens and colleagues demonstrated greater activation of the SMG during lipreading when compared to static images of a face (Ruytjens et al., 2006). Taken together, one could predict that L2 ASL learners might show greater activation in the STS and SMG during audiovisual processing after sign language exposure.

In the present study, we tested the hypothesis that exposure to a novel sign language would change monolingual audiovisual processing. In a two-time point longitudinal study, we scanned the brains of hearing second language (L2) ASL learners before significant exposure to ASL. We tested their ability to categorize words in the scanner based on whether the word started with a sound produced on the lips or not. After approximately 13 weeks, or one semester, of sign language exposure, we scanned their brains again while they performed the same task. Self-rating measures of the use of co-sign speech (i.e., mouthings) and lipreading were collected at both time points. A multiple regression analysis was performed in order to investigate whether changes in mouthings or lipreading could explain any of the differences in brain activation before and after sign language exposure. A separate behavioral study was 
conducted after the completion of the MRI study wherein hearing controls were tested behaviorally on the same task outside of the scanner to ensure that improvement was not merely practice effects.

It was predicted that behaviorally there would be a significant increase in audiovisual phoneme categorization accuracy after one semester of instruction for L2 ASL learners. No significant change in categorization performance for hearing nonsigners was predicted. At the neural level, greater STS activation after one semester of instruction was predicted given that greater audiovisual processing was expected. The enhanced audiovisual processing is predicted to elicit greater SMG activation at T1 because better audiovisual processing would facilitate more phonetic-phonological processing of the lexical stimuli. Affirmative findings would inform our understanding of how sign language exposure can enhance spoken language processing.

\section{Results}

\section{Behavioral Results with Post-Hoc Hearing Nonsigner Controls}

In order to examine whether there were behavioral changes in audiovisual ability and to rule out possible practice effects, hearing nonsigner controls also performed the behavioral task outside of the scanner. Participants' accuracy scores and response times (RT) across the trials in identifying the location of the speech sound (on the lips or elsewhere) were calculated and compared using a repeated-measures analysis of variance (ANOVA), with time (T0 vs. T1) as the within-subjects factor and Group (L2 vs. Controls) as the between-subjects factor. Age was not considered as a covariate because it did not correlate with performance $\left[r_{T 0}=0.133, p=\right.$ 
$\left.0.454 ; r_{T 1}=-0.065, p=0.716\right]$ and a mean difference of three years in young adults would not be expected to have an impact on audiovisual processing.

There was no main effect of time when collapsing across both groups, such that participants had similar accuracy at T0 $(M=84.8 \%, S E=2.9)$ as compared to T1 $(M=90.2 \%$, $S E=2.3)\left[F(1,32)=2.606, p=0.116, \eta_{\mathrm{p}}{ }^{2}=0.075\right]$. Response times also did not differ between $\mathrm{T} 0(M=1792 \mathrm{~ms}, S E=466)$ and $\mathrm{T} 1(M=1878 \mathrm{~ms}, S E=450)\left[F(1,32)=0.694, p=0.441, \eta_{\mathrm{p}}{ }^{2}\right.$ $=0.021]$. No main effect of group was observed for accuracy scores $[F(1,32)=0.005, p=$ $\left.0.942, \eta_{\mathrm{p}}{ }^{2}=0\right]$; however, there was for response times $\left[F(1,32)=9.075, p<0.01, \eta_{\mathrm{p}}{ }^{2}=0.221\right]$. L2 ASL learners were generally slower $(M=2022 \mathrm{~ms}, S E=495)$ than hearing nonsigner controls $(M=1644 \mathrm{~ms}, S E=421)$. There was a trending interaction between time and group for accuracy scores $\left[F(1,32)=3.205, p=0.083, \eta_{\mathrm{p}}{ }^{2}=0.091\right]$, but not for response times $[F(1,32)=$ $\left.0.684, p=0.414, \eta_{\mathrm{p}}^{2}=0.021\right]$.

Given that there was an a priori hypothesis that L2 learners would improve over time and there was a trending interaction effect of time and group, planned paired t-tests were performed comparing each time point for each group. Only accuracy rates were compared between $\mathrm{T} 0$ and $\mathrm{T} 1$ given that there were no significant differences in RTs. A significant increase in audiovisual phoneme classification was observed for the hearing ASL learners from the first $(M=81.96 \%, S E=5.3)$ to the second time point $(M=93.31 \%, S E=0.9), t(16)=2.202$, $p<0.05$. However, there was no significant increase in accuracy between the first $(M=87.6 \%$, $S E=9.4)$ and the second $(M=87.0 \%, S E=18.2)$ testing for the control group, $t(16)=0.138, p$ $=0.89$. This suggests that the ASL learners were better able to determine the location of a speech sound in their L1 after only a semester of ASL, and this was not likely due to practice effects. Additionally, despite a qualitative difference at T0 between the ASL learners' 
performance $(81.9 \%)$ and the controls' $(87.6 \%)$, there was no significant difference in baseline performance $[t(32)=0.971, p=0.339]$, which indicates that differences between groups is likely restricted to sign language exposure and not other baseline factors.

Changes in L2 Neural Audiovisual Processing

$$
\text { <Figure } 1 \text { about here> }
$$

\section{TO Audiovisual Processing}

Table 1 Brain regions activated at T0 $(p<0.001 ; k \geq 25)$

\begin{tabular}{lcrrr}
\hline \multirow{2}{*}{ Cerebral regions (Brodmann Area) } & \multirow{3}{*}{ No. of voxels } & \multicolumn{3}{c}{ Coordinates (mm) } \\
& & \multicolumn{1}{c}{$y$} & \multicolumn{1}{c}{$z$} \\
\hline L superior temporal gyrus (22) & 25 & -60 & -44 & 8 \\
R superior temporal gyrus (22) & 25 & 48 & -30 & 0 \\
L middle temporal gyrus (21) & 42 & -50 & -14 & -10 \\
L superior parietal lobule (5) & 44 & -24 & -66 & 46 \\
L inferior occipital gyrus (18) & 84 & -42 & -82 & -14 \\
R temporal pole (38) & 26 & 56 & 6 & -18
\end{tabular}

According to the fMRI results, before any substantial exposure to sign language, hearing English speakers showed activation in few regions for audiovisual words greater than fixation. The activation was primarily located in the temporal lobe (see Figure 1 and Table 1). Specifically, the left and right superior temporal gyri (BA 22), left middle temporal gyrus (BA 21), and right temporal pole (38) were activated. Significant activation was found in the left superior parietal lobule (BA 5) and left inferior occipital gyrus (BA 18) as well.

\section{T1 Audiovisual Processing}

Table 2 Brain regions activated at $T 1(p<0.001 ; k \geq 25)$

\begin{tabular}{lccccc}
\hline \hline Cerebral regions (Brodmann Area) & No. of voxels & \multicolumn{3}{c}{$\begin{array}{c}\text { Coordinates } \\
\text { (mm) }\end{array}$} \\
& & $x$ & $y$ & $z$ \\
\hline L supramarginal gyrus (40) & 337 & -66 & -24 & 24
\end{tabular}




$\begin{array}{lcrrr}\text { R supramarginal gyrus (40) } & 28 & 42 & -52 & 58 \\ \text { L temporal pole (38) } & 35 & -42 & 2 & -18 \\ \text { R superior temporal pole (38) } & 32 & 38 & 10 & -22 \\ \text { L superior temporal gyrus (22) } & 111 & -60 & -54 & 10 \\ \text { L inferior parietal lobule (7) } & 33 & -48 & -40 & 44 \\ \text { R inferior parietal lobule (7) } & 25 & -34 & -58 & 40 \\ \text { R superior parietal lobule (7) } & 26 & 34 & -50 & 60 \\ \text { R superior temporal gyrus (22) } & 294 & 62 & -46 & 6 \\ \text { R posterior cingulate gyrus (31) } & 106 & 4 & -20 & 34 \\ \text { L anterior insula (13) } & 241 & -46 & -10 & 4 \\ \text { R primary somatosensory cortex (3) } & 30 & 56 & -26 & 56 \\ \text { R inferior frontal gyrus (47) } & 89 & 54 & 18 & -6 \\ \text { R primary motor cortex (4) } & 71 & 38 & -28 & 60 \\ \text { L anterior cingulate gyrus (24) } & 25 & 0 & 32 & 30 \\ \text { R cingulate gyrus (24) } & 66 & 8 & 22 & 44 \\ \text { L pyramis } & 46 & -8 & -78 & -34 \\ \text { R caudate body } & 107 & 16 & 0 & 20 \\ \text { R putamen } & 30 & 34 & -10 & 4 \\ \text { L pulvinar (19) } & 52 & -14 & -30 & 1 \\ \text { L cuneus (17) } & 3387 & -8 & -80 & 18 \\ \text { R cuneus (17) } & 32 & 10 & -82 & 44 \\ \text { L middle occipital gyrus (19) } & 82 & -54 & -70 & -8 \\ \text { R lingual gyrus (19) } & 58 & 18 & -74 & -12\end{array}$

During time point 2 the same participants showed greater activation across the cortex as well as sub-cortically (see Figure 1 and Table 2). The occipital cortex was extensively activated during this task, such that the left and right cuneus (BA 17), right lingual gyrus (BA 19), and left middle occipital gyrus (BA 19) were activated. The temporal lobe was also engaged with activation in the left and right temporal pole (BA 38), left and right superior temporal gyrus (BA 22), left insula (BA 13), and right inferior frontal gyrus (BA 47). The supramarginal gyrus (BA 40), bilateral inferior and superior parietal lobule (BA 40 and 7, respectively), and primary motor (BA 4) and somatosensory cortex (BA 3) showed significant clusters. The left anterior cingulate cortex (BA 24) was significant as well. Subcortical regions were significantly 
engaged, including the right putamen, right caudate body, and the left pulvinar. Cerebellar activation in the left pyramis was also observed.

T0 versus $T 1$

Table 3 Brain regions activated greater in T1 than TO $(p<0.001 ; k \geq 25)$

\begin{tabular}{lcrrr}
\hline \multirow{2}{*}{ Cerebral regions (Brodmann Area) } & \multirow{3}{*}{ No. of voxels } & \multicolumn{3}{c}{ Coordinates (mm) } \\
& & $x$ & \multicolumn{1}{c}{$y$} \\
\hline L supramarginal gyrus (40) & 31 & -58 & -28 & 22 \\
R supramarginal gyrus (40) & 46 & -22 & 26 \\
L lingual gyrus (19) & 42 & -14 & -62 & 0 \\
L anterior insula (13) & 40 & -44 & -10 & 4 \\
R inferior frontal gyrus (47) & 46 & 48 & 18 & 2 \\
R supplemental motor area (6) & 27 & 4 & -10 & 56
\end{tabular}

When the two time points were directly compared using a paired t-test, L2 learners of ASL were found to show greater activation of the supramarginal gyrus (BA 40) bilaterally when categorizing phonemes in an audiovisual task. Additionally, learners showed significant clusters in the left lingual gyrus (BA 19), left insula (BA 13), right inferior frontal gyrus (BA 47), and the right supplemental motor area (BA 6).

Multiple Regression Analysis

$<$ Figure 2 about here>

$<$ Figure 3 about here >

Table 4 Brain regions correlated with composite score differences $(p<0.005 ; k \geq 15)$

\begin{tabular}{lcccc}
\hline \hline \multirow{2}{*}{ Cerebral regions (Brodmann Area) } & \multirow{2}{*}{ No. of voxels } & \multicolumn{3}{c}{ Coordinates (mm) } \\
& & $x$ & $y$ & $z$ \\
\hline L supramarginal gyrus (40) & 19 & -58 & -46 & 30 \\
L inferior temporal gyrus (20) & 16 & -52 & -68 & -4 \\
L primary motor cortex (4) & 25 & -40 & -20 & 62
\end{tabular}


In an attempt to explore the relationship between the activation observed at $\mathrm{T} 1$ and the increase in mouthing/lipreading, the composite mouthing-lipreading scores were calculated at each time point and a difference score (i.e., T1 - T0) was derived. At the beginning of the semester, the participants had an average composite score of 1.90 (1.03). After one semester ( $\sim 13$ weeks) of exposure to sign language, their average composite score significantly increased to $3.35(1.36), t(16)=3.777, p<0.01$. The difference scores across $\mathrm{T} 0$ and $\mathrm{T} 1$ were included in a multiple regression analysis of activation at $\mathrm{T} 1$. The thresholds were set at an uncorrected pvalue of 0.005 with an extent threshold of 15 voxels in order to explore possible correlations. Activation in the left SMG (BA 40), inferior temporal gyrus (BA 20), and primary motor cortex (BA 4) during the audiovisual categorization task was positively correlated with the participants' change in composite mouthing-lipreading ratings. Small volume correction was also performed on the supramarginal cluster, as it was an a priori region of interest that was predicted to show change. The results revealed that the correlation between the composite mouthing-lipreading score and activation of the region was significant when correcting for multiple comparisons using FWE $(p<0.05)$.

\section{General Discussion}

The goal of the present study was to investigate whether exposure to sign language enhances audiovisual processing. In the present study participants viewed audiovisual English words and classified the words based on the labial visibility of the initial phoneme. Behavioral results indicated that L2 learners of sign language marginally improved phoneme classification after only 13 weeks of sign language exposure when compared to hearing nonsigner controls. Brain activation also significantly increased to audiovisual words after sign exposure. 
Specifically, there was a significant increase in activation in the left and right supramarginal gryi, which may be indicative of greater phonological processing. Increases in co-sign speech (i.e., mouthing) and lipreading during sign language production may be involved in enhanced audiovisual word processing as a multiple regression analysis revealed a significant correlation in the left supramarginal gyrus (BA 40). Together, these results indicate that the divergence between articulatory systems allows for simultaneous mouth and hand movements, which may act as visual training for spoken language perception.

\section{Bimodal Bilingualism as Multisensory Training}

Evidence for improved performance on a behavioral audiovisual categorization task after only 13 weeks of sign language exposure contributes to our understanding of multimodal language processing and experienced-induced neuroplasticity. Ostensibly, the L2 ASL learners in the present study did not undergo any overt audiovisual or multisensory training in their first semester of ASL courses. Nevertheless, learners performed approximately $11 \%$ better on the audiovisual categorization task at the end than at the beginning of the semester. One possible explanation for their improved performance is that their new multimodal linguistic environment acted as multisensory training. Novice learners of sign languages often look at the mouth of the signing interlocutor during sign communication (Emmorey, Thompson, \& Colvin, 2008). Prolonged eye gaze to a signer's mouth despite any actual vocalizations is thought to arise due to the beginning learner's attempt to capture English mouthing. This falls in line with the fact that often hearing bimodal bilinguals will mouth English translations of the signs they are producing (i.e., code-blending; Capek, Campbell, \& Woll, 2008; Davis, 1990; Emmorey et al., 2008). Additionally, sign languages use the mouth for grammatical inflection, lexical 
disambiguation, and gesturing (Bickford \& Fraychineaud, 2008; Boyes-Braem \& SuttonSpence, 2001; Sandler, 2009). The ecological requirements of acquiring a sign language as a second language reinforce lipreading and mouthing abilities. In turn, their general cognitive architecture is restructured insofar as their ability to perceive phonemic contrasts in their native spoken language improves. However, no improvements were shown in reaction times, which would suggest that L2 ASL learners do not improve automatic audiovisual processing, but rather general audiovisual accuracy. Support for this potential relationship between ASL L2 learning and lipreading is the increased brain activation in the supramarginal gyrus, a region implicated in phonological processing and has also been correlated with increased frequency in mouthing and lipreading.

Another potential explanation for the current findings is that their improved performance was due to task repetition; participants are performing the same task twice. However, the increase in behavioral performance that accompanies task repetition typically elicits neural adaptation effects, or a reduction in brain activation at T1 (Grill-Specter, Henson, \& Martin, 2005; Schacter, Wig, \& Stevens, 2007). Here greater activation at T1 compared to T0 was observed. Therefore, neural activation patterns appear to not support task repetition priming as an explanation for the results presented. Instead the increase in activation at T1 might be indicative of increased ability to process audiovisual language or change in strategy. That is, increased T1 activation is in and of itself evidence for better phonological and articulatory (see below) processing, which could be caused by a change in strategy due to the ASL exposure. Additionally, behavioral data indicate that beginning learners of a sign language might draw attention to the mouth of a signing interlocutor and require co-sign mouthings in order to comprehend recently-learned lexical items in sign language. As such, overreliance on the mouth 
during sign communication as well as immersion in a visual environment may impact their spoken language audiovisual processing.

\section{Supramarginal Gyrus in Phonological Processing}

After 13 weeks of sign language exposure learners showed greater activation in the supramarginal gyrus during audiovisual phoneme categorization compared to their pre-exposure baseline. Additionally, increased activation of the supramarginal gyrus during the second time point may indicate that the learners were better able to extract the phonological form during audiovisual processing. The supramarginal gyrus has been implicated in phonological processing in numerous studies (Demonet et al., 1992; Hartwigsen et al., 2010; Sliwinska et al., 2012; Stoeckel et al., 2009). Hartwigsen et al. (2010) demonstrated disruption to phonological judgments (but not semantic judgments) when transcranial magnetic stimulation was applied over the left supramarginal gyrus. This is consistent with a number of other findings that have implicated the left supramarginal gyrus in phonological processing in short-term memory (Romero, Walsh, \& Papagno, 2005) and visual word recognition (Sliwinska et al., 2012; Stoeckel et al., 2009).

The task used in the current study was to determine whether the word presented had an initial sound that was produced labially or not. The relative difficulty of the task can be seen in the accuracy score at the first time point (82\%). It is likely that due to the noise of the scanner participants had to rely not just on auditory aspects of the stimuli but also on the mouthing of the speaker. Increased activation at time point two (T1) in the anterior insula and supramarginal gyrus was observed. The anterior insula has long been linked to articulatory processes (Wise et al., 1999) while, as mentioned above, the supramarginal gyrus has been linked to phonological 
processing. Finding increased activation in these two regions after ASL training suggests that participants may have not only realized the need to use mouthing information to perform the task but have accessed similar articulatory codes in order to accurately respond to the stimuli. In fact, a recent study has shown the hearing individuals with sign language experience have increased grey matter volume in the left insular cortex, which was attributed to dependence on lipreading (Allen, Emmorey, Bruss, \& Damasio, 2008). Together this suggests that the ASL learners learned to focus on mouthing and to extract information that was important for ASL perception as well and English perception.

While both the anterior insula and the supramarginal gyrus showed increased activation at time point two, only the supramarginal activation was found to be correlated with the increased frequency of lipreading by the ASL students. This correlation revealed that the greater the increase in lipreading the greater the activation in the supramarginal gyrus. This may suggest that the increase in the use of mouthing information may more strongly impact phonological processing, in this case the extraction of phonetic information from an audiovisual stimulus.

\section{Audiovisual Integration}

Given that there was significant activation in the supramarginal gyrus, which was correlated with increased use of mouthing and lipreading, it is surprising that areas responsible for audiovisual integration did not also show differential activation. Audiovisual integration is thought to occur in the posterior portion of the superior temporal sulcus (Campbell, 2008; Hocking \& Price, 2008; Noesselt et al., 2007). In fact, posterior superior temporal areas are especially attuned to mouth movements that mirror speech and its auditory spatiotemporal 
dynamics (Campbell, 2008). In the present study, learners showed activation in superior temporal regions at both time points. Such activation is indicative of adequate audiovisual integration; in fact, without such integration their behavioral accuracy scores would be even lower. Despite activation in areas associated with audiovisual integration, the difference between the two time points was non-significant. Furthermore, superior temporal areas were not correlated with mouthing-lipreading difference scores. These findings suggest that the learners in the present study were able to adequately integrate multimodal speech information and their integration abilities were not augmented by sign language experience. Rather, increased activation in the supramarginal gyrus, which was also correlated with mouthing-lipreading scores, indicates that sign language exposure offers a unique opportunity to focus on the phonological form presented on the lips and not the integration of auditory and visual information. During sign language learning, the majority of the sign input that was given to the leaners did not contain auditory information, as their professors were native deaf signers and did not use spoken English in the classroom. The lack of auditory information during acquisition, however, does not preclude borrowed English mouthings from their instructors nor does it preclude code-blending from their hearing classmates. Therefore, the learning environment may explain differential activation in the supramarginal gyrus but not in superior temporal regions after sign language exposure.

\section{Conclusion and Limitations}

The results presented here lend more support for previous findings that show that learning a second language has implications for the processing of the first language. Understanding these effects is important because it has been argued that the divergence between 
signed and spoken language modalities prevents any significant interaction between native and second language phonological systems (Giezen \& Emmorey, 2015; Shook \& Marian, 2012; Williams \& Newman, 2015), which might also reduce neural changes to the native language network and neurocognitive abilities. Although the present study was able to demonstrate an emerging relationship between sign language exposure and audiovisual processing, there are limitations to the interpretation of the present data.

The L2 ASL learners performed the task inside of the MRI scanner and the hearing nonsigners were outside of the scanner; one might ascribe the present results to the presence of scanner noise for the L2 learners. It might be argued that these participants required greater visual cues due to the masked auditory information, which in turn the L2 learners implemented a more robust lipreading strategy compared to the hearing nonsigners. However, the effect observed is an increase in the use of lipreading after ASL training, which does not explain why these participants failed to adopt this lipreading strategy at T0. In other words, we would not predict an enhancement in audiovisual processing based solely on sound level differences because the potential differences in and outside of the scanner should have remained constant across time points, which would not advantage nor disadvantage either group. Additionally, both groups were tested to ensure they could hear the stimuli regardless of environment. Given the nature of the task, these particular participants ostensibly lacked advanced metaphonological knowledge where they could categorize bilabial and non-bilabial sounds auditorily; rather both groups most likely depended on visual information to categorize bilabial sounds, which would negate differences in auditory presentation. Therefore, the improvement in audiovisual processing over time for the L2 ASL learners is not likely due to any environmental variables. However, future studies will be needed to investigate whether this is the case. 
There was a significant difference between groups in terms of reaction times. The L2 learner group was slower overall compared to the hearing nonsigners. It is difficult to determine the source of this difference. It is unlikely that slower reaction times represented a speedaccuracy trade-off because there was no interaction between group and time. As such, the reaction times remained constantly slower in the L2 group across time. If it were a true speedaccuracy trade-off, we would expect better performance by the L2 group at both time points. However, the L2 group performed worse that than control group at T0. It is more likely that the scanner environment (e.g., using a hand paddle instead of a keyboard) slowed down response times for the L2 group.

The results presented suggest that the improvements in the audiovisual processing of L2 ASL learners are not due to practice effects but to ASL training. It is possible, however, that practice effects could arise at the neural level without manifesting behaviorally. The present study is not able to differentiate possible practice effects at the neural level. Future studies will be needed in order to rule out this possibility as well. Furthermore, we demonstrated that learning a sign language may improve lipreading as indicated by the increase in self-reported lipreading. It is not clear whether the same is true when learning another spoken language or if this is a unique property of sign language learning. Additional studies that include a spoken language L2 group are necessary to address that question.

\section{Experimental Procedure}

\section{Participants}

Seventeen $($ male $=7)$ hearing L2 learners of American Sign Language participated in both the behavioral and neuroimaging tasks, who were a subset of participants from Williams, 
Darcy, and Newman (2015, in press). All L2 ASL learners were right-handed. Their mean age was 19.6 years (1.5 years). Learners were recruited from introductory ASL courses at Indiana University. These 17 learners were the students who volunteered to come back for the first two time points (i.e., at baseline and after one semester) during our first wave of longitudinal data collection. $^{2}$

Seventeen $($ male $=1)$ hearing nonsigners participated in the post-hoc study as a control group in the behavioral study. Sixteen nonsigners were right-handed $($ left $=1)$ with a mean age of 23.1 years (4 years). These participants were to make sure that any behavioral improvements seen in the hearing learners were due to ASL experience and not practice effects. However, the control participants did not participate in the neuroimaging study.

All participants reported no previous exposure to sign language before. All participants reported English as their first language. There was a significant difference in age between the groups $[t(16)=3.655, \mathrm{p}=0.002]$, where the age difference was approximately 3.5 years. All participants gave written consent to perform the experimental tasks, which was approved by the Indiana University Institutional Review Board.

\section{Experimental Design}

L2 ASL learners performed the experimental task in an MRI scanner during the first week of classes (T0) and during the last week of classes (T1). Time points one and two were approximately 13 weeks apart, with students having approximately 43.9 (1.12) hours of ASL instruction. Participants completed a background questionnaire at each time point that included information regarding their exposure to a sign language. L2 ASL learners also rated their use of co-sign speech and lipreading during sign language production and perception. They were asked, 1) "how often do you mouth English words while signing?"; 2) "how many signs are 
accompanied with English mouthing when communicating?"; and 3) "how often do you currently use lipreading to communicate?". They ranked their responses on a 7-point Likert scale from " $1=$ Never" to "7 = Always" for the first and third questions and from " $1=$ None" to “7 = Every Sign" for the second. An average composite score was derived to get an overall measure of their mouthing (i.e., co-sign speech) and lipreading during sign communication.

The hearing nonsigners controls performed the experimental task twice outside of the scanner with approximately 4 weeks between T0 and T1. Although the time between test and retest differed from the L2 ASL learners, the difference would actually favor the hearing nonsigners controls since any practice effects would be greater with less time between repetitions. Both groups performed the same categorization task only twice regardless of whether it was inside or outside of the scanner.

The behavioral classification task was composed of 30 trials and was approximately 9 minutes in duration. Participants viewed a native English speaker saying various English words. The speaker's full face and torso were shown in front of a blue-gray backdrop, which were recorded using a Sony HDR-CX220 video camera and edited using Adobe Premiere Pro software. All stimuli were high frequency monomorphemic words from various word classes (i.e., nouns, verbs, adjectives). All stimuli were constrained to four phonemes in length. Half of the stimuli started labial sounds (i.e., bilabial and labio-dental; e.g., /m/, /p/, /b/, /f/, /v/); the other half started with non-labial sounds (i.e., alveolar, velar, and liquid; e.g., /k/, /g/, /s/, /t/, /l/). Additionally, the second set of stimuli did not contain bilabials throughout the word (except for come; refer to the Appendix for a table of the stimuli).

The functional task was presented in an event-related design. For each trial a 500millisecond fixation point was presented before the video appeared. Each stimulus video varied 
in duration $(M=1593.33, S D=2.53 \mathrm{~ms})$ and was followed by a jittered interstimulus interval (range $=4000-8000, M=6000 \mathrm{~ms}$ ). Participants were told to press the right index finger for words that began with a visible phoneme (i.e., labial sounds) and the left index finger for words that began with phonemes not visible on the lips (i.e., alveolar, velar, or liquid). They were instructed to make their responses as quickly and accurately as possible. In addition to the ISI, a 30 second fixation was presented at the beginning of the run and was used as a baseline.

The L2 ASL learners performed this task within the MRI scanner, whereas the hearing nonsigners controls performed the task outside of the scanner as a post-hoc group comparsion. For both instances, the task was controlled using PsychoPy (Peirce, 2007) on a 13-inch MacBook Pro, where the same visual aspect was controlled and the distance from the participant's eye and screen was approximately 12 inches for both groups. The auditory stimuli were presented over headphones at a comfortable listening level. An audio-only test video (not included in the experimental session) was played to both groups and the subjects were asked to repeat the contents and adjusting the volume level accordingly. This was done to assure that both the L2 ASL learners and the hearing nonsigners controls had access to the auditory information despite the possible sound-level differences inside and outside of the scanner and that they were not relaying on visual information. Button responses were recorded using an inhouse MRI-safe button box for the L2 ASL learners and via the computer keyboard for the hearing nonsigners controls.

For the ASL learners, they were also tested in a number of other behavioral tests both inside and outside of the scanner as part of a larger longitudinal study (see Williams et al., in press). The order of behavioral and scanner sessions were randomly chosen to prevent any cross-experimental confounds; nevertheless, none of the other behavioral tasks required 
audiovisual processing that may act as a training task for these participants. The tasks within the scanner always followed the same order wherein the English audiovisual task presented in this manuscript always came first, preceding any other phonetic discrimination tasks in Spanish or ASL (see Williams et al., 2015). Thus, it is unlikely that the order to tasks or sessions would have influenced the results herein.

\section{Imaging Parameters}

L2 ASL learners underwent 2 scans using a 32-channel coil and a Siemens 3 Tesla TIM Trio MRI scanner. The first scan was an anatomical T1-weighted scan used to co-register functional images. An MPRAGE sequence (160 sagittal slices; FOV=256 mm, matrix $=256 \times 256, T R=2300 \mathrm{~ms}, \mathrm{TE}=2.91 \mathrm{~ms}, \mathrm{TI}=900 \mathrm{~ms}$, flip angle $=9^{\circ}$, slice thickness $=1 \mathrm{~mm}$,

resulting in $1-\mathrm{mm} \times 1-\mathrm{mm} \times 1-\mathrm{mm}$ voxels) was used. The second scan was the experimental functional multiband EPI scan (59 axial slices using the following protocol: field of view=220 $\mathrm{mm}$, matrix $=128 \times 128$, iPAT factor $=2, \mathrm{TR}=2000 \mathrm{~ms}, \mathrm{TE}=30 \mathrm{~ms}$, flip angle $=60^{\circ}$, slice thickness $=2 \mathrm{~mm}, 0$ gap).

\section{Data analysis}

Data was analyzed using SPM8 (Wellcome Imaging Department, University College, London, UK, freely available at http://fil.ion.ucl.ac.uk/spm). During preprocessing images were corrected for slice acquisition timing, and resampled to $2 \times 2 \times 2 \mathrm{~mm}^{3}$ isovoxels, spatially smoothed with a Gaussian filter with a $4 \mathrm{~mm}$ kernel. All data was high-pass filtered at 1/128 $\mathrm{Hz}$ to remove low-frequency signals (e.g., linear drifts). Motion correction was performed and motion parameters incorporated into the design matrix. Each participant's anatomical scan was 
aligned and normalized to the standardized SPM8 T1 template and then fMRI data was coregistered to anatomical images. At the individual level, statistical analysis was performed using the General Linear Model and Gaussian random fields. The video onsets and durations were entered as regressors in the model (Friston et al., 1995). The first level contrasts were activation to the audiovisual stimuli (AV) minus the fixation baseline at the beginning of the experiment. For the second level (random effects) analysis on group data, paired t-tests were performed between time points on the AV - Fixation contrasts. To correct for multiple comparisons (Forman et al., 1995), the dimensions and smoothing parameter of processed data were entered into AFNI's AlphaSim program. With a voxel-wise $p<0.001$, clusters greater than 25 voxels were considered significant after 5000 Monte Carlo iterations with a corrected alpha $<0.05$. A multiple regression was also performed in order to find activation that is correlated with the learners' mouthing-lipreading scores. As an exploratory analysis in order to investigate possible contributions to any significant results, a multiple regression analysis was carried out with an uncorrected voxel-wise $p<0.005$ and a cluster extent threshold of 15. An uncorrected threshold was used in order to determine whether there were any positive correlations with the mouthinglipreading score. Small volume correction $\left(p_{F W E}<0.05\right)$ for any significant activation in either STS or SMG was performed in order to correct for multiple comparisons for those a priori regions. 


\section{Acknowledgements}

Supported by the National Science Foundation (NSF) Integrative Graduate Education and Research Training Program in the Dynamics of Brain-Body-Environment Systems at Indiana University (JTW) and the NSF Graduate Research Fellowship (JTW). Funding also provided by the Indiana University Imaging Research Facility Brain Scan Credit Program (JTW, ID, \& SDN).

\section{Endnote}

${ }^{1}$ Sign language lexical glosses are denoted in small capital letters.

${ }^{2}$ In Williams et al. (2015) there were a total of 39 monolinguals at pre-exposure baseline (T0), 33 of which were enrolled in ASL courses. Of those 39, there were 17 who came back after one semester of sign language exposure (T1) during our first wave of longitudinal data collection. Those were the 17 included herein. Of those 17, only 12 came back for the last time point (i.e., two semesters after baseline, T2) and were included in Williams et al. (in press). 


\section{References}

Allen, J. S., Emmorey, K., Bruss, J., \& Damasio, H. (2008). Morphology of the insula in relation to hearing status and sign language experience. The Journal of Neuroscience, 28(46), 11900-11905.

Bickford, J. A., \& Fraychineaud, K. (2006). Mouth morphemes in ASL: A closer look. In Sign Languages: spinning and unraveling the past, present and future. Papers from the Ninth Theoretical Issues in Sign Language Research Conference, Florianopolis, Brazil.

Boyes-Braem, P., Sutton-Spence, R., \& Leiden, R. (Eds.). (2001). The hands are the head of the mouth: The mouth as articulator in sign languages. Signum.

Campbell, R. (2008). The processing of audio-visual speech: empirical and neural bases.

Philosophical Transactions of the Royal Society B: Biological Sciences, 363(1493), 1001-1010.

Capek, C., Campbell, R., \& Woll, B. (2008). The bimodal bilingual brain: fMRI investigations concerning the cortical distribution and differentiation of signed language and speechreading. Rivista di Psicolinguistica Applicata, 3, 97-112.

Davis, J. (1990). Linguistic transference and interference: Interpreting between English and ASL. Sign language research: Theoretical issues, 308-321.

Demonet, J. F., Chollet, F., Ramsay, S., Cardebat, D., Nespoulous, J. L., Wise, R., Rascol, A., \& Frackowiak, R. (1992). The anatomy of phonological and semantic processing in normal subjects. Brain, 115(6), 1753-1768. 
Draganski, B., Gaser, C., Busch, V., Schuierer, G., Bogdahn, U., \& May, A. (2004).

Neuroplasticity: changes in grey matter induced by training. Nature, 427(6972), 311312.

Emmorey, K., Borinstein, H. B., Thompson, R., \& Gollan, T. H. (2008). Bimodal bilingualism. Bilingualism: Language and Cognition, 11(01), 43-61.

Emmorey, K., Grabowski, T., McCullough, S., Ponto, L. L., Hichwa, R. D., \& Damasio, H. (2005). The neural correlates of spatial language in English and American Sign Language: a PET study with hearing bilinguals. Neuroimage, 24(3), 832-840.

Emmorey, K., Thompson, R., \& Colvin, R. (2009). Eye gaze during comprehension of American Sign Language by native and beginning signers. Journal of Deaf Studies and Deaf Education, 14(2), 237-243.

Forman, S. D., Cohen, J. D., Fitzgerald, M., Eddy, W. F., Mintun, M. A., \& Noll, D. C. (1995). Improved assessment of significant activation in functional magnetic resonance imaging (fMRI): use of a cluster-size threshold. Magnetic Resonance in medicine, 33(5), 636647.

Friston, K. J., Holmes, A. P., Poline, J. B., Grasby, P. J., Williams, S. C. R., Frackowiak, R. S., \& Turner, R. (1995). Analysis of fMRI time-series revisited. Neuroimage, 2(1), 45-53.

Giezen, M. R., \& Emmorey, K. (2015). Language co-activation and lexical selection in bimodal bilinguals: Evidence from picture-word interference. Bilingualism: Language and Cognition, 1-13.

Girin, L., Schwartz, J. L., \& Feng, G. (2001). Audio-visual enhancement of speech in noise. The Journal of the Acoustical Society of America, 109(6), 3007-3020. 
Grill-Spector, K., Henson, R., \& Martin, A. (2006). Repetition and the brain: neural models of stimulus-specific effects. Trends in Cognitive Sciences, 10(1), 14-23.

Hartwigsen, G., Baumgaertner, A., Price, C. J., Koehnke, M., Ulmer, S., \& Siebner, H. R. (2010). Phonological decisions require both the left and right supramarginal gyri. Proceedings of the National Academy of Sciences, 107(38), 16494-16499.

Hocking, J., \& Price, C. J. (2008). The role of the posterior superior temporal sulcus in audiovisual processing. Cerebral Cortex, 18(10), 2439-2449.

Jones, Ō. P., Green, D. W., Grogan, A., Pliatsikas, C., Filippopolitis, K., Ali, N., Lee, H.L., Ramsden, S., Gazarian, K., Prejawa, S., Seghier, M.L., \& Price, C. J. (2012). Where, when and why brain activation differs for bilinguals and monolinguals during picture naming and reading aloud. Cerebral Cortex, 22(4), 892-902.

Kovelman, I., Baker, S. A., \& Petitto, L. A. (2008). Bilingual and monolingual brains compared: an fMRI investigation of syntactic processing and a possible "neural signature" of bilingualism. Journal of Cognitive Neuroscience, 20(1), 153-169.

Lehmann, S., \& Murray, M. M. (2005). The role of multisensory memories in unisensory object discrimination. Cognitive Brain Research, 24(2), 326-334.

Leonard, M. K., Ramirez, N. F., Torres, C., Travis, K. E., Hatrak, M., Mayberry, R. I., \& Halgren, E. (2012). Signed words in the congenitally deaf evoke typical late lexicosemantic responses with no early visual responses in left superior temporal cortex. The Journal of Neuroscience, 32(28), 9700-9705.

Lidestam, B., \& Beskow, J. (2006). Visual phonemic ambiguity and speechreading. Journal of Speech, Language, and Hearing Research, 49(4), 835-847. 
Lovelace, C. T., Stein, B. E., \& Wallace, M. T. (2003). An irrelevant light enhances auditory detection in humans: a psychophysical analysis of multisensory integration in stimulus detection. Cognitive brain research, 17(2), 447-453.

Massaro, D. W., \& Simpson, J. A. (2014). Speech perception by ear and eye: A paradigm for psychological inquiry. Psychology Press.

McGurk, H., \& MacDonald, J. (1976). Hearing lips and seeing voices. Nature, 264, 746-748.

MacSweeney, M., Woll, B., Campbell, R., McGuire, P. K., David, A. S., Williams, S. C., Suckling, J., Calvert, G.A., \& Brammer, M. J. (2002). Neural systems underlying British Sign Language and audio-visual English processing in native users. Brain, 125(7), 15831593.

MacSweeney, M., Campbell, R., Woll, B., Brammer, M. J., Giampietro, V., David, A. S., Calvert, G.A., \& McGuire, P. K. (2006). Lexical and sentential processing in British Sign Language. Human Brain Mapping, 27(1), 63-76.

MacSweeney, M., Capek, C. M., Campbell, R., \& Woll, B. (2008). The signing brain: The neurobiology of sign language. Trends in Cognitive Sciences, 12(11), 432-440.

McCarthy, G., Puce, A., Belger, A., \& Allison, T. (1999). Electrophysiological studies of human face perception. II: Response properties of face-specific potentials generated in occipitotemporal cortex. Cerebral Cortex, 9(5), 431-444.

Mechelli, A., Crinion, J. T., Noppeney, U., O'Doherty, J., Ashburner, J., Frackowiak, R. S., \& Price, C. J. (2004). Neurolinguistics: structural plasticity in the bilingual brain. Nature, 431(7010), 757-757. 
Mei, L., Xue, G., Lu, Z.L., Chen, C., Zhang, M., He, Q., Wei, M., \& Dong, Q. (2014). Learning to read words in a new language shapes the neural organization of the prior languages, Neuropsychologia, 65, 156-168.

Navarra, J., \& Soto-Faraco, S. (2007). Hearing lips in a second language: visual articulatory information enables the perception of second language sounds. Psychological Research, 71(1), 4-12.

Noesselt, T., Rieger, J. W., Schoenfeld, M. A., Kanowski, M., Hinrichs, H., Heinze, H. J., \& Driver, J. (2007). Audiovisual temporal correspondence modulates human multisensory superior temporal sulcus plus primary sensory cortices. The Journal of Neuroscience, 27(42), 11431-11441.

Pelphrey, K. A., Mitchell, T. V., McKeown, M. J., Goldstein, J., Allison, T., \& McCarthy, G. (2003). Brain activity evoked by the perception of human walking: Controlling for meaningful coherent motion. The Journal of Neuroscience, 23(17), 6819-6825.

Petitto, L. A., Zatorre, R. J., Gauna, K., Nikelski, E. J., Dostie, D., \& Evans, A. C. (2000). Speech-like cerebral activity in profoundly deaf people processing signed languages: implications for the neural basis of human language. Proceedings of the National Academy of Sciences, 97(25), 13961-13966.

Romero, L., Walsh, V., \& Papagno, C. (2006). The neural correlates of phonological short-term memory: a repetitive transcranial magnetic stimulation study. Journal of Cognitive Neuroscience, 18(7), 1147-1155. 
Ruytjens, L., Albers, F., Van Dijk, P., Wit, H., \& Willemsen, A. (2006). Neural responses to silent lipreading in normal hearing male and female subjects. European Journal of Neuroscience, 24(6), 1835-1844.

Sandler, W. (2009). Symbiotic symbolization by hand and mouth in sign language. Semiotica, 2009(174), 241-275.

Schacter, D. L., Wig, G. S., \& Stevens, W. D. (2007). Reductions in cortical activity during priming. Current Opinion in Neurobiology, 17(2), 171-176.

Schlaug, G., Forgeard, M., Zhu, L., Norton, A., Norton, A., \& Winner, E. (2009).

Training-induced Neuroplasticity in Young Children. Annals of the New York Academy of Sciences, 1169(1), 205-208.

Shook, A., \& Marian, V. (2012). Bimodal bilinguals co-activate both languages during spoken comprehension. Cognition, 124(3), 314-324.

Sliwinska, M. W., Khadilkar, M., Campbell-Ratcliffe, J., Quevenco, F., \& Devlin, J. T. (2012). Early and sustained supramarginal gyrus contributions to phonological processing. Frontiers in Psychology, 3.

Stoeckel, C., Gough, P. M., Watkins, K. E., \& Devlin, J. T. (2009). Supramarginal gyrus involvement in visual word recognition. Cortex, 45(9), 1091-1096.

Strelnikov, K., Rosito, M., \& Barone, P. (2011). Effect of audiovisual training on monaural spatial hearing in horizontal plane. PloS one, 6(3), e18344.

Sumby, W. H., \& Pollack, I. (1954). Visual contribution to speech intelligibility in noise. The Journal of the Acoustical Society of America, 26(2), 212-215. 
Summerfield, Q. (1987). Comprehensive account of audio-visual speech perception. In Hearing by the eye: The psychology of lip-reading (B.E. Dodd and R.E. Campbell, eds.). Lawrence Erlbaum Associates, Inc.

Vigliocco, G., Perniss, P., \& Vinson, D. (2014). Language as a multimodal phenomenon: implications for language learning, processing and evolution. Philosophical Transactions of the Royal Society B: Biological Sciences, 369(1651), 20130292.

Williams, J.T., Darcy, I., Newman, S.D. (in press). Modality-specific processing precedes amodal linguistic processing during L2 sign language acquisition: a longitudinal study. Cortex, 1 - 19. doi: 10.1016/j.cortex.2015.11.015

Williams, J.T., Darcy, I., \& Newman, S.D. (2015). Modality-independent neural mechanisms for novel phonetic processing. Brain Research, 1620, 107-115. doi: 10.1016/j.brainres.2015.05.014

Williams, J., \& Newman, S. (2015) Interlanguage dynamics and lexical networks in nonnative L2 signers of ASL: cross-modal rhyme priming. Bilingualism: Language and Cognition, $1-18$.

Wise, R. J. S., Greene, J., Büchel, C., \& Scott, S. K. (1999). Brain regions involved in articulation. The Lancet, 353(9158), 1057-1061.

Wright, T. M., Pelphrey, K. A., Allison, T., McKeown, M. J., \& McCarthy, G. (2003). Polysensory interactions along lateral temporal regions evoked by audiovisual speech. Cerebral Cortex, 13(10), 1034-1043. 


\section{Figure Captions}

Fig. 1. Activation from a whole-brain analysis at time points one (T0) and two (T1) is plotted.

Fig. 2. Results from a multiple regression analysis using mouthing-lipreading difference scores with activation at $\mathrm{T} 1$ (uncorrected $p<0.005, k \geq 15$ ).

Fig. 3. Scatter plot of the T1 activation in the left supramarginal gyrus as a function of the change in mouthing-lipreading scores from T0 to T1 (i.e., T1 - T0). By using a change in mouthing-lipreading scores, Figure 3 demonstrates that those who used more mouthing or lipreading with time also showed greater activation in the LSMG at T1 $\left(p_{F W E}<0.05\right)$. 


\section{Appendix}

A1. Stimuli for phoneme classification task.

\begin{tabular}{|c|c|c|c|}
\hline \multicolumn{2}{|c|}{ Frontal Phonemes } & \multicolumn{2}{|c|}{ Back Phonemes } \\
\hline band & /bænd/ & kids & /kidz/ \\
\hline born & /born/ & land & /lænd/ \\
\hline bank & /bæyk/ & gold & /gold/ \\
\hline bend & /bend/ & last & /læst/ \\
\hline belt & /belt/ & come & /kəm/ \\
\hline film & /film/ & sold & /sold/ \\
\hline park & /park/ & card & /kard/ \\
\hline post & /post/ & lost & /lpst/ \\
\hline plan & /plæn/ & sink & $/ \operatorname{sink} /$ \\
\hline mark & /mark/ & size & /sajz/ \\
\hline mute & /mjut/ & told & /told/ \\
\hline pump & /pəmp/ & coast & /kost/ \\
\hline mint & /mint/ & star & /star/ \\
\hline vent & /vent/ & tent & /tent/ \\
\hline plus & /pləs/ & send & /send/ \\
\hline
\end{tabular}


TO
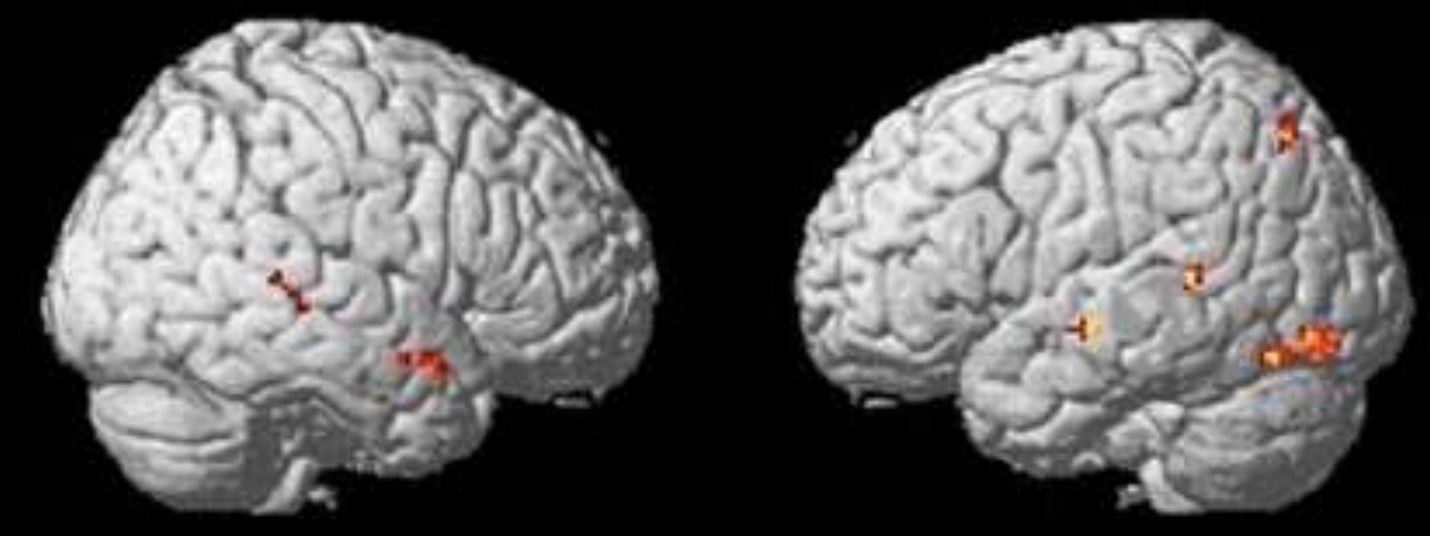

T1
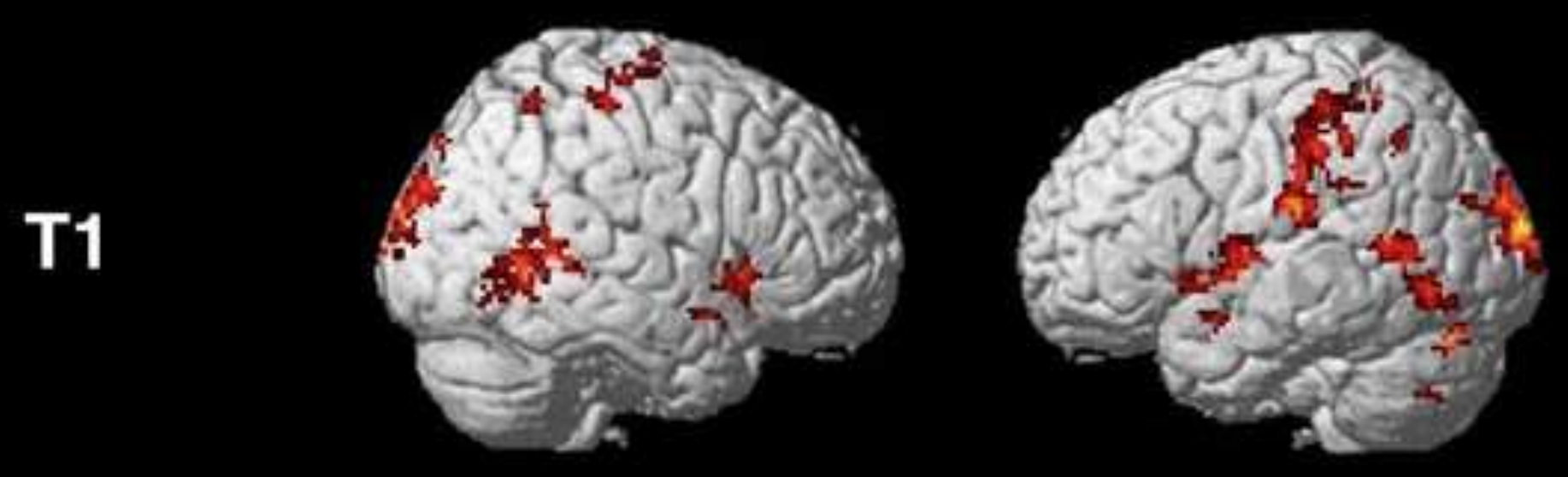

T1 > T0
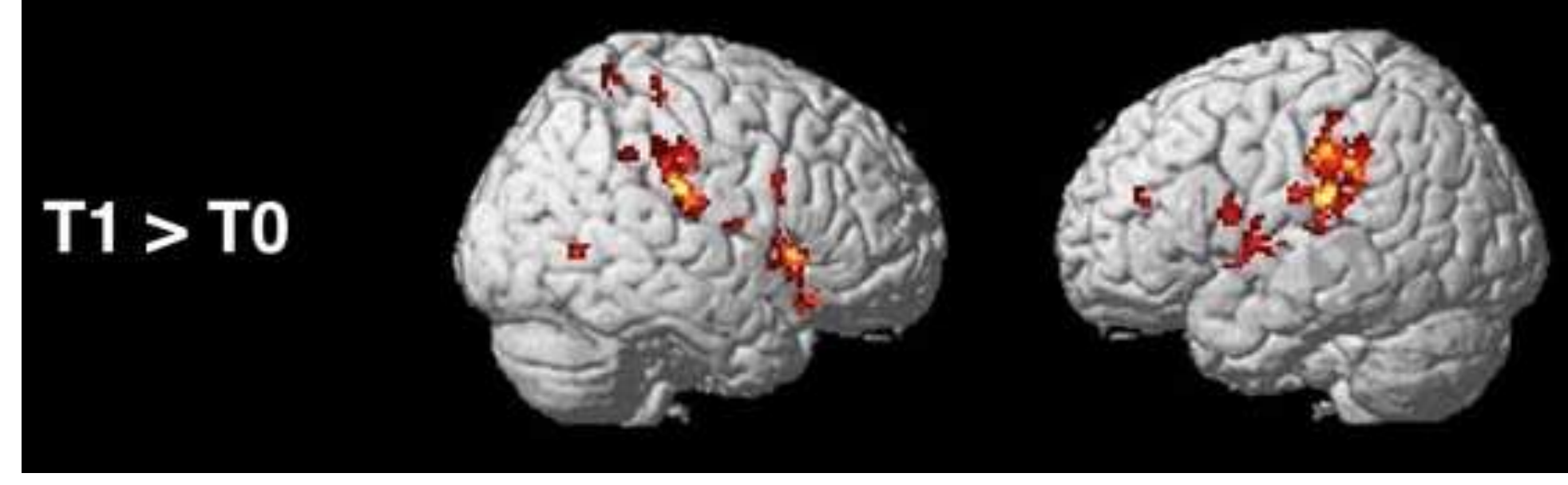

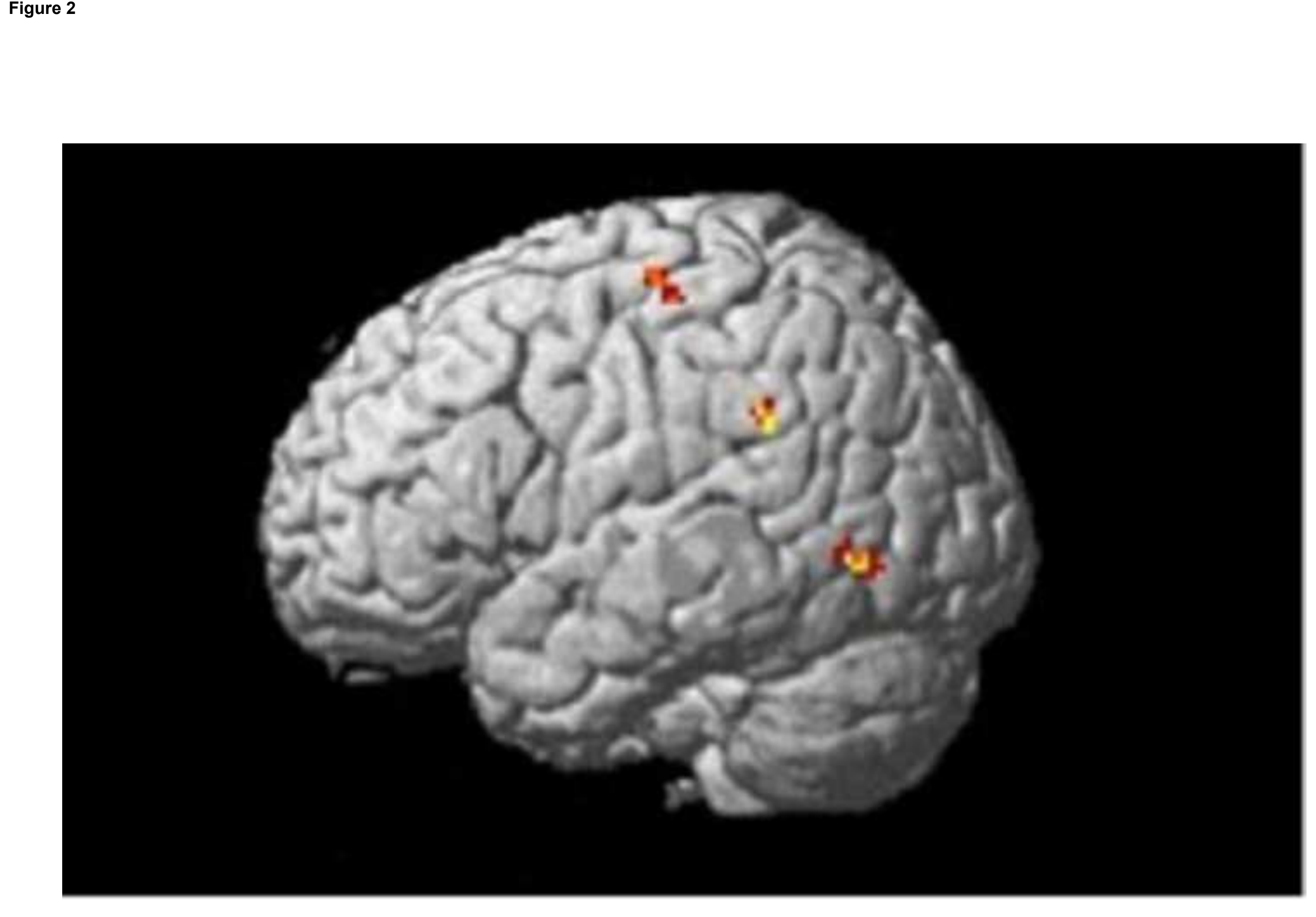

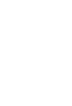
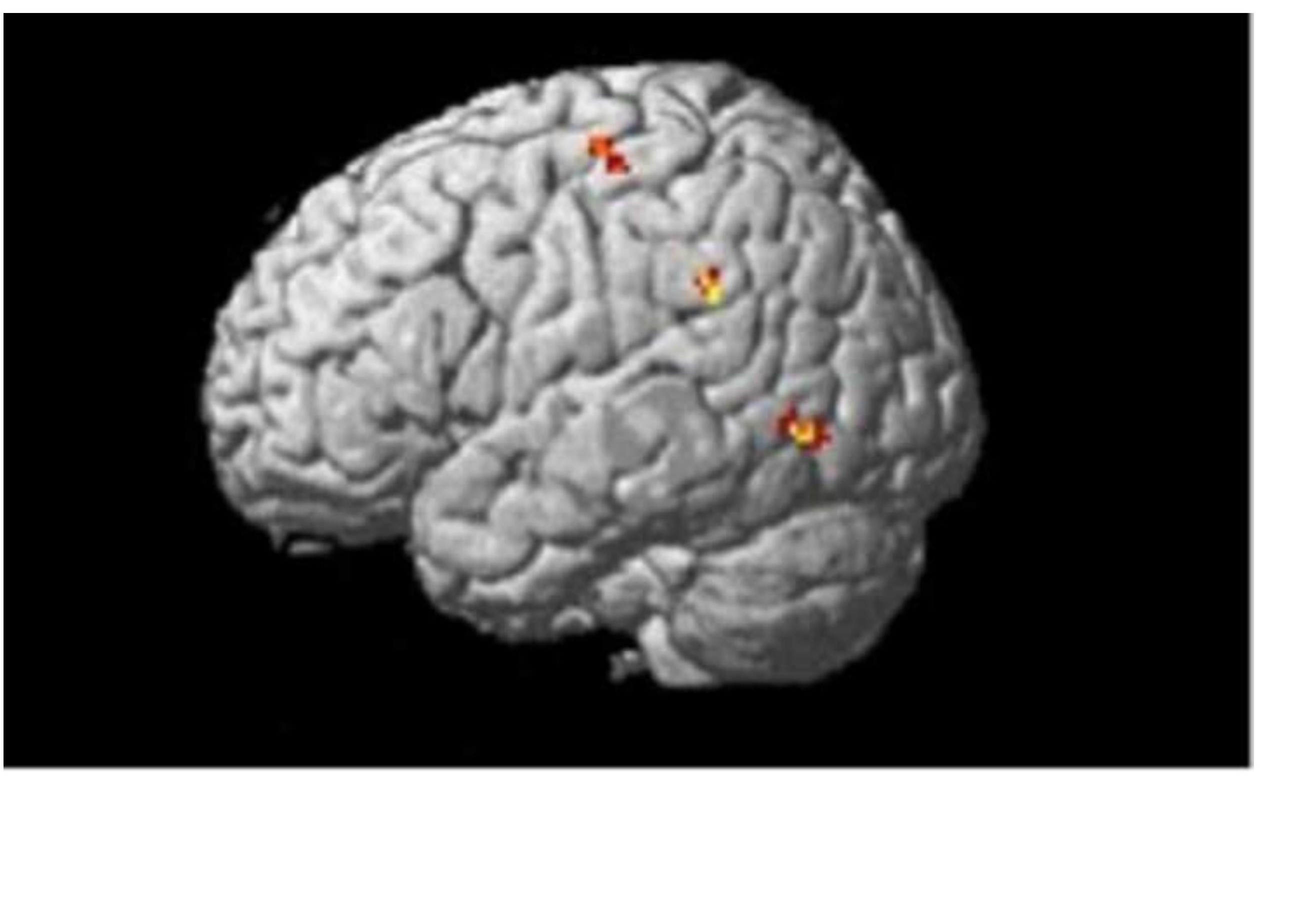


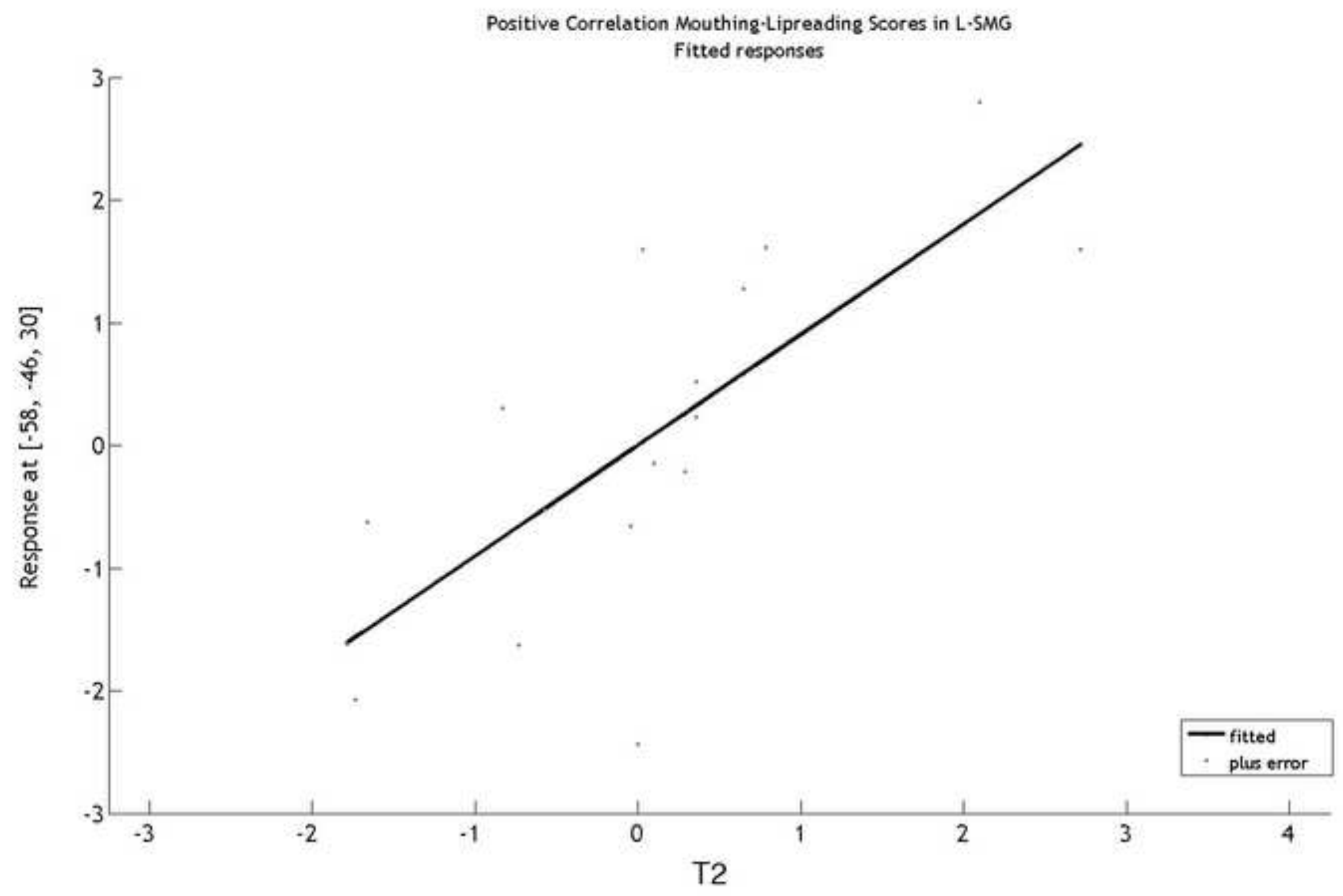

